\title{
Biobased Synthesis of Gold Nanoparticles by Methanobactin
}

\author{
Jia-ying Xin ${ }^{1,2, a^{*}}$, Shuai Zhang ${ }^{1, b}$ \\ ${ }^{1}$ Key Laboratory for Food Science \& Engineering, Harbin University of Commerce, Harbin 150076, \\ People's Republic of China \\ ${ }^{2}$ State Key Laboratory for Oxo Synthesis \& Selective Oxidation, Lanzhou Institute of Chemical \\ Physics, Chinese Academy of Sciences, Lanzhou 730000, People's Republic of China \\ axinjiayingvip@163.com.cn, ${ }^{b}$ roson@163.com
}

Keywords: Methanobactin; Methanotrophs; Au (III) reduction; Gold nanoparticles.

\begin{abstract}
Preparation of gold nanoparticles with a narrow size distribution has enormous importance in nanotechnology. Methanobactin $(\mathrm{Mb})$ is a copper-binding small peptide that appears to function as an agent for $\mathrm{Cu}$ sequestration and uptake in methanotrophs. Mb can also bind and catalytically reduce $\mathrm{Au}$ (III) to $\mathrm{Au}(0)$. In the presence of hydroquinone as a electron donator, $\mathrm{Mb}$ can catalyze $\mathrm{Au}(\mathrm{III})$ reduce to $\mathrm{Au}(0)$ and yield gold nanoparticles continuously. In this paper, a quantitative assay method for the content of $\mathrm{Mb}$ has been used. Continuous reduction of $\mathrm{Au}$ (III) by $\mathrm{Mb}$ can be achieved by using hydroquinone as the reducing agent. Effect of $\mathrm{pH}$, reaction temperature, reaction time on Mb-mediated synthesis of gold nanoparticles was studied. The optimal conditions of gold nanoparticles synthesis were as follows: the optimum $\mathrm{pH}$ value was 5.1 , the optimum reaction temperature was $40{ }^{\circ} \mathrm{C}$, the optimum reaction time was 15-20min. The gold nanoparticles are extremely stable and can resist aggregation, even after several months.
\end{abstract}

\section{Introduction}

Methanotrophs are a group of Gram-negative eubacteria that utilize methane as the sole energy and carbon source ${ }^{[1]}$. In the first step of carbon assimilation by methanotrophs, methane is oxidized to methanol by Methane Monooxygenase (MMO). MMO exists in both the iron-containing soluble form (soluble methane monooxygenase, sMMO) and the copper-containing membrane-bound particulate form (particulate methane monooxygenase, pMMO) ${ }^{[2]}$. Copper requirements are higher for methanotrophic bacteria. Since the expression of copper containing particulate MMO (pMMO) depends on copper availability ${ }^{[3]}$. One way that methanotrophic bacteria meet their high copper requirement is by the biosynthesis and release of high affinity copper binding compounds called Methanobactin $(\mathrm{Mb})$. Methanobactin $(\mathrm{Mb})$ is a copper-binding small peptide that appears to function as an agent for copper detoxification, sequestration and uptake in methanotrophs. $\mathrm{Mb}$ has been identified in the extracellular fractions of both Methylosinus trichosporium OB3b ${ }^{[4,5]}$ and Methylococcus capsulatus Bath ${ }^{[6]}$. Mb is composed of a tetrapeptide, a tripeptide, and several unusual moieties. The copper-loaded $\mathrm{Mb}$ from Methylosinus trichosporium OB3b is a $1217 \mathrm{Da}$ peptide that binds and coordinates copper by two thionyl imidazolate moieties in a N2S2 distorted tetrahedral arrangement ${ }^{[4]}$. $\mathrm{Mb}$ can also bind and reduce $\mathrm{Au}(\mathrm{III})$ to $\mathrm{Au}(0)^{[5]}$. In the previous paper, we have demonstrated a facile $\mathrm{Mb}$-mediated synthetic route to prepare gold nanoparticles ${ }^{[7]}$. In this paper, the effect of $\mathrm{pH}$, reaction temperature and reaction time on the formation of gold nanoparticles were further studied.

\section{Materials and methods}

Culture of methanotrophs. Methylosinus trichosporium 3011 was obtained from the Institute of Microbiology and Virology. Methanotrophs were cultivated with mineral salts medium ${ }^{[7]}$. Cultivation was carried out in a $250 \mathrm{~mL}$ closed vial with $50 \mathrm{~mL}$ mineral salts medium on a shaker at $150 \mathrm{rpm}$. and 
$30{ }^{\circ} \mathrm{C}$. Methane was added periodically by establishing a partial vacuum in the flask and backfilling with methane and air (1:10). The gas phase methane level was maintained at about $10 \%(\mathrm{~V} / \mathrm{V})$ by exchanging the headspace volume three times per day.

Culture conditions for Mb production. A volume $(5 \mathrm{~mL})$ of Methylosinus trichosporium 3011 cultures from mid exponential growth phase was used to inoculate mineral salts medium $(50 \mathrm{~mL})$ with various concentration of methanol in a $250 \mathrm{ml}$ closed conical flask. Methanol was added after sterilization of the mineral salts medium. Cultures were grown on air or mixture of methane and air $(1: 10, \mathrm{~V} / \mathrm{V})$ at $30^{\circ} \mathrm{C}$ with shaking $(150 \mathrm{rpm})$. The cultures were grown to stationary phase for $\mathrm{Mb}$ production. Copper ( II ) was omitted from the medium.

Preparation of $\mathbf{M b}$. Mb from the spent medium of Methylosinus trichosporium 3011 was isolated as previously described for Methylococcus capsulatus Bath by Choi et al. ${ }^{[6]}$ and Methylosinus trichosporium OB3b by Kim et al. ${ }^{[10]}$. The cells were removed by centrifugation at $10,000 \times \mathrm{g}$ for 30 min. The supernate was loaded onto a $2.5 \times 20-\mathrm{cm}$ Diaion HP-20 column (Mitsubishi Chemical Holdings, Tokyo, Japan). The bound $\mathrm{Mb}$ was washed with two column volumes of $\mathrm{H}_{2} \mathrm{O}$ and eluted with $40 \%$ methanol: $60 \% \mathrm{H}_{2} \mathrm{O}$. The eluant was lyophilized for concentration and storage. The freeze-dried samples following chromatography on Diaion HP-20 columns were the source of Mb used in this study.

Results and discussion. According to Yoon et al ${ }^{[8]}$, we have developed a method to determine $\mathrm{Mb}$ quantitatively by using its high affinity for copper based on the comparative exchange of copper (II) from an indicator dye CAS. The combination of CAS, HDTMA and copper ratio of 1.0:8.0:0.7 proved to be most advantageous for Mb detection ${ }^{[9]}$. In this paper, this method has been used to determine $\mathrm{Mb}$ concentration. As shown in Figure 1, the changes in the absorbance spectra of the CAS after the release of copper were detected with EDTA and Mb. The spectrum of the CAS-Cu-HDTMA complex clearly showed a sharp absorbance peaks at $605 \mathrm{~nm}$ as a function of copper removal from $\mathrm{CAS}$. Both $\mathrm{Mb}$ and EDTA exhibit a linear dependence of the absorbance at $605 \mathrm{~nm}$ versus concentration of the chelator. So the EDTA concentration was chosen as an equivalent of $\mathrm{Mb}$ content for detection of $\mathrm{Mb}$ concentration in solution. A working curve between the change of absorbance of $\mathrm{CAS}-\mathrm{Cu}$ and the EDTA concentration was established (data not shown).

$\mathrm{Mb}$ can bind $\mathrm{Cu}$ (II) ions, and reduce them to $\mathrm{Cu}$ (I) ions and stabilizes the resulting $\mathrm{Cu}$ (I) ions using a mechanism that is not well understood. It has been reported that $\mathrm{Mb}$ from Methylosinus trichosporium OB3b contains

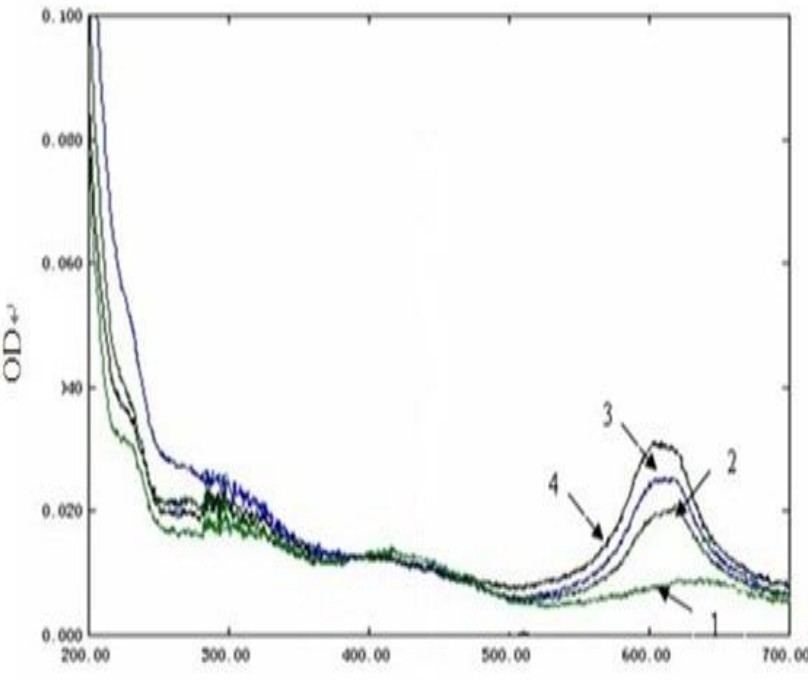

Figure. 1 The absorbance spectra of the CAS reagent
1: CAS+HDTMA;
2: CAS+HDTMA+ $\mathrm{CuSO}_{4}+\mathrm{EDTA}$;
3: $\mathrm{CAS}+\mathrm{HDTMA}+\mathrm{CuSO}_{4}+\mathrm{mb}$;
4: $\mathrm{CAS}+\mathrm{HDTMA}+\mathrm{CuSO}_{4}$ two oxazolone rings and two thiol groups that are directly involved in binding $\mathrm{Cu}$ (I) ions. $\mathrm{Mb}$ can also be able to bind and reduce a number of other metals, including gold. Researchers have determined that gold in the 3+ oxidation state, $\mathrm{Au}$ (III), can be reduced to its zero oxidation state, $\mathrm{Au}(0)$ at or below ratios of one $\mathrm{Au}$ (III) per $\mathrm{Mb}$ molecule. At ratios of $\mathrm{Au}$ (III) to $\mathrm{Mb}$ above one to one, $\mathrm{Mb}$ binds and catalytically reduces $\mathrm{Au}(\mathrm{III})$ to $\mathrm{Au}(0)$ with the concomitant production of gold nanoparticles ${ }^{[5]}$. We 
have reported a facile $\mathrm{Mb}$-mediated one-step synthetic route to prepare monodispersed gold nanoparticles. Continuous reduction of $\mathrm{Au}$ (III) by $\mathrm{Mb}$ can be achieved by using hydroquinone as the reducing agent ${ }^{[7]}$.

In this paper, this approach that $\mathrm{Mb}$ binds and catalytically reduces $\mathrm{Au}$ (III) to $\mathrm{Au}(0)$ has been used for the synthesis of gold nanoparticles. Continuous reduction of gold by $\mathrm{Mb}$ was achieved with hydroquinone as electron donor. As been shown in Figure 2, Figure 3 and Figure 4, it has been found
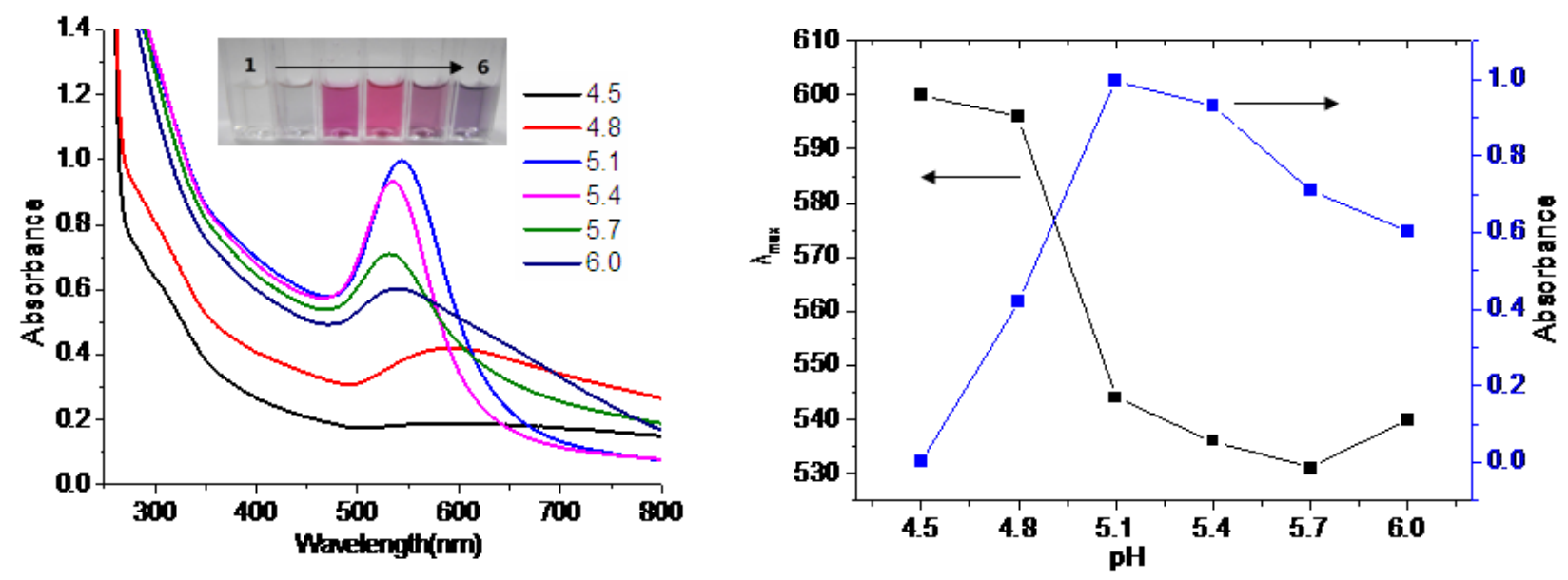

Figure. 2 The effect of $\mathrm{pH}$ on the formation of gold nanoparticles.
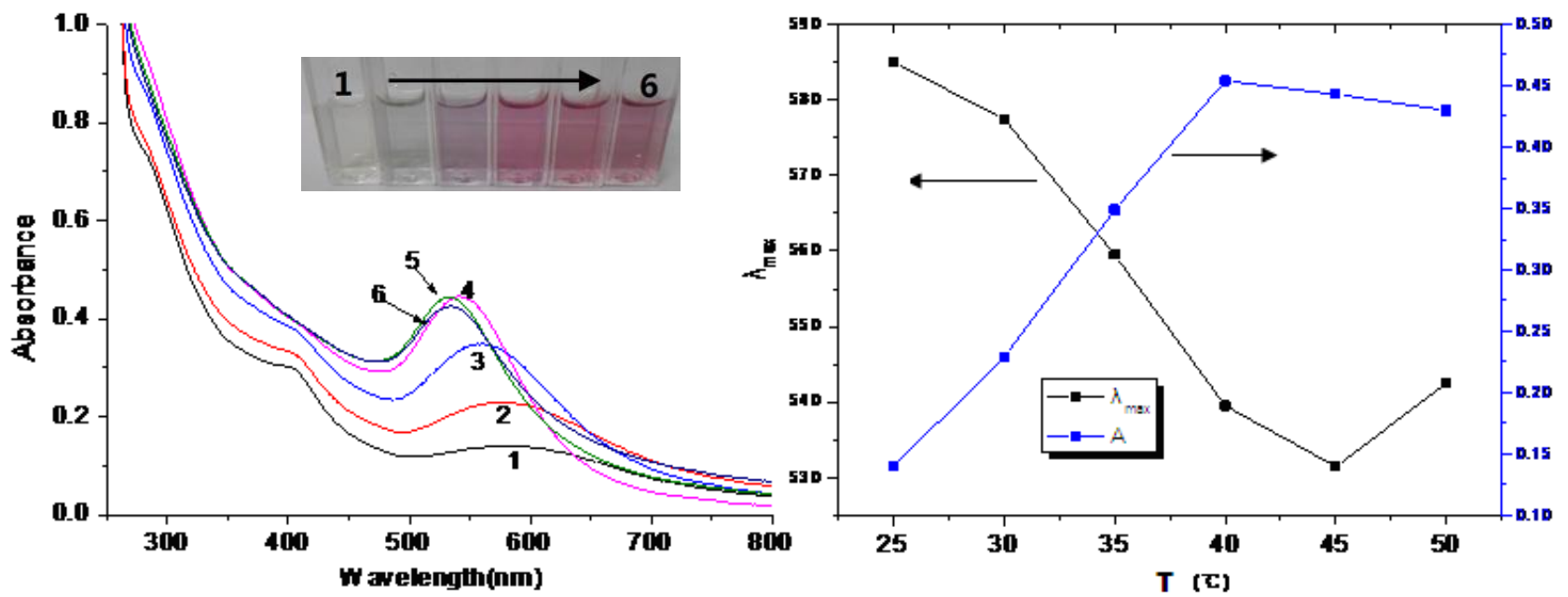

Figure. 3 The effect of reaction temperature on the formation of gold nanoparticles.

1: $25^{\circ} \mathrm{C} ; 2: 30^{\circ} \mathrm{C} ; 3: 35^{\circ} \mathrm{C} ; 4: 40^{\circ} \mathrm{C} ; 5: 45^{\circ} \mathrm{C} ; 6: 50^{\circ} \mathrm{C}$
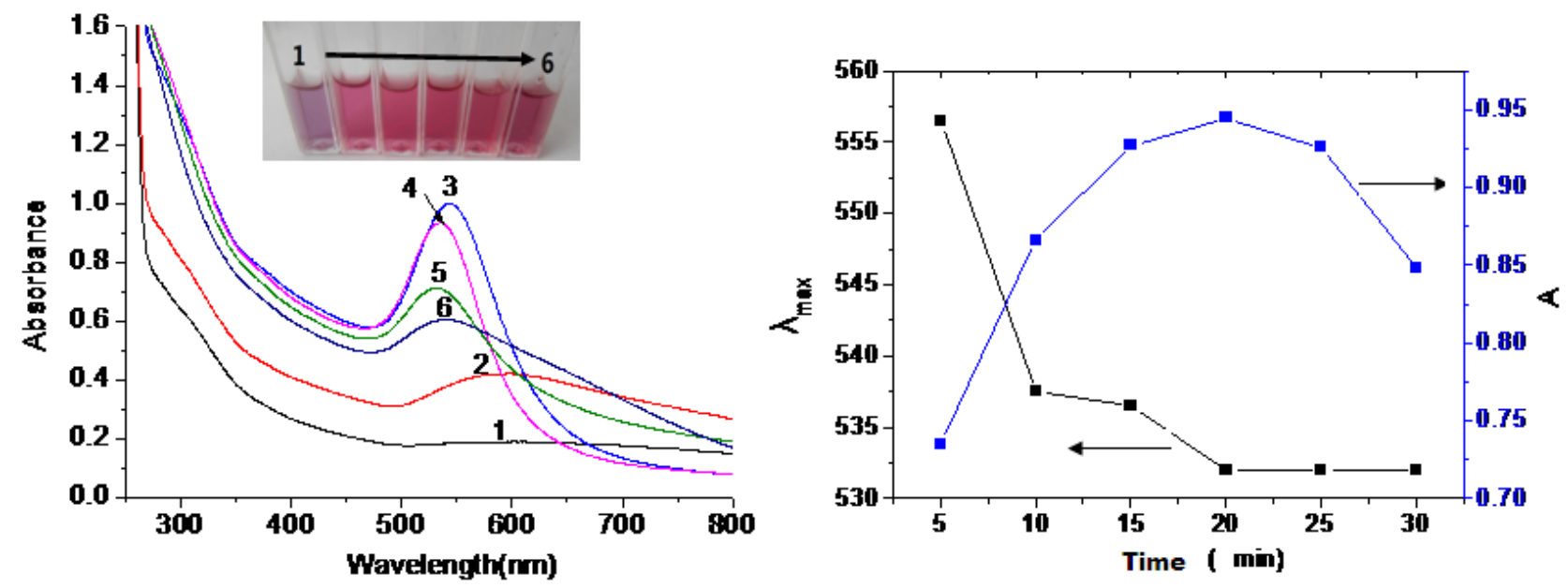

Figure. 4 The effect of reaction time on the formation of gold nanoparticles.

1:5min; 2:10min; 3:15min; 4: 20min; 5:25min; 6: 30min 
that the optimum $\mathrm{pH}$ value was 5.1 ; the optimum reaction temperature was $40{ }^{\circ} \mathrm{C}$; the optimum reaction time was 15-20min. Gold nanoparticles can be seen with the naked eye, as they turn a gold solution from yellow/gold to a deep cranberry/red. The gold nanoparticles also display a characteristic absorbance of approximately $540 \mathrm{~nm}-550 \mathrm{~nm}$ on absorption spectra. Also, the gold nanoparticles are extremely stable and can resist aggregation, even after several months.

\section{Acknowledgements}

The authors thank the Scientific Research Fund of Heilongjiang Province (GC 13C111), the Scientific Research Fund of Heilongjiang Provincial Education Department (11551z007) and the Heilongjiang Provincial Funds for Distinguished Young Scientists (JC201106) for support.

\section{References}

[1] R. S. Hanson, T. E. Hanson, Methanotrophic bacteria. Microbiol. Rev. 60 (1996), 439-471

[2] A. S. Hakemian, A. C. Rosenzweig, The biochemistry of methane oxidation. Annu Rev Biochem.76 (2007), 223-241

[3] E. Kulczycki, D. A. Fowle, C. Knapp, D. W. Graham, J. A. Roberts, Methanobactin-promoted dissolution of $\mathrm{Cu}$-substituted borosilicate glass. Geobiology. 5(2007), 251-263

[4] H. J. Kim, D. W. Graham, A. A. DiSpirito, M. A. Alterman, N. Galeva, C. K. Larive, D. Asunskis, P. M. A. Sherwood, Methanobactin, a copper-aquisition compound from methane oxidizing bacteria. Science. 305(2004), 1612-1615

[5] D. W. Choi, Y. S. Do, C. J. Zea, M. T. McEllistrem,; S. W. Lee,; J. D. Semrau,; N. L. Pohl, C. J. Kisting,; L. L. Scardino,; S. C. Hartsel,; Spectral and thermodynamic properties of Ag(I), Au(III), $\mathrm{Cd}(\mathrm{II}), \mathrm{Co}(\mathrm{II}), \mathrm{Fe}(\mathrm{III}), \mathrm{Hg}(\mathrm{II}), \mathrm{Mn}$ (II), $\mathrm{Ni}(\mathrm{II}), \mathrm{Pb}(\mathrm{II}), \mathrm{U}(\mathrm{IV})$, and $\mathrm{Zn}$ (II) binding by methanobactin from Methylosinus trichosporium OB3b. J. Inorg. Biochem., 100 (2006), 2150-2161

[6] D. W. Choi, W. E. Antholine, Y. S. Do, J. D. Semrau, C. J. Kisting, R. C. Kunz, D. Campbell, V. Rao, S. C. Hartsel, A. A. DiSpirito, Effect of methanobactin on the activity and electron paramagnetic resonance spectra of the membrane-associated methane monooxygenase in Methylococcus capsulatus Bath. Microbiology .151(2005), 3417-3426

[7] J. Y. Xin, D. D. Chen, L. X. Zhang, K. Lin, H. C. Fan, Y. Wang, C. G. Xia, Methanobactin-Mediated One-Step Synthesis of Gold Nanoparticles, Int. J. Mol. Sci. 14 (2013), 21676-21688;

[8] S. Yoon, S. M. Kraemer, A. A. DiSpirito, J. D. Semrau, An assay for screening microbial cultures for chalkophore production. Environ Microbiol Reports. 2(2010), 295-303

[9] J. Y. Xin, L. X. Zhang, D. D. Chen, S. Zhang, Y. Wang, C. G. Xia, Study on the production and species-specificity of methanobactin, Journal of Chemical and Pharmaceutical Research, 5(2013), $168-176$

[10] H. J. Kim, N. Galeva, C. K. Larive et al., M. Alterman, D. W. Graham, Purification and physical-chemical properties of methanobactin: a chalkophore from Methylosinus trichosporium OB3b. Biochemistry. 44 (2005), 5140-5148 